\title{
Stellar Population Synthesis in the Bulge of our Galaxy
}

\author{
ANNIE C. ROBIN \\ Observatoire de Besançon, BP 1615, F-25010 Besançon cedex, France
}

\begin{abstract}
Using a model of population synthesis well tuned to reproduce the disc and halo populations of the Galaxy, we show how to set constrains on the bulge population parameters using magnitude and colour distribution in 4 directions near the bulge. This first analysis result in a bulge with a disc-like LF but a density law much steeper in the center.
\end{abstract}

Key words: Milky Way Galaxy - galaxy modeling - stellar populations

\section{Introduction}

Connecting observable distributions in the Galaxy to the main processes they come from is basically a multivariate problem for which we have developed a synthetic approach of Galaxy modeling (hereafter referred to as the Besançon model, Robin and Crézé, 1986, Bienaymé et al., 1987). The relation between the bulge, the disc, the thick disc and the halo should be addressed using all kinds of observational parameters (photometric as well as astrometric). In this first attempt we compare magnitude and colour distributions in the visible and the near-infrared in 4 directions close to the galactic bulge in order to put constraints on its stellar content.

\section{Galaxy model}

Our specific approach is based on assumptions directly derived from current pictures of galactic evolution. Observational parameters like velocities, space distribution and metallicities are all driven by the age parameter. Age distributions come from a specific evolution model (Rocca-Volmerange et al., 1981), while the disc density laws have been self consistently constrained with the whole potential of the galaxy using Boltzmann and Poisson equations and an age-velocity dispersion relation (Bienaymé et al., 1987).

\section{Comparison with bulge data}

Three directions at longitude $10^{\circ}$ and latitude around $-10^{\circ},-20^{\circ}$ and $-30^{\circ}$ have been observed by Rodgers \& Harding (1989) (hereafter $\mathrm{RH}$ ) giving a good sample to study the gradient of populations near the bulge. While Ruelas-Mayorga \& Teague (hereafter RT) produces $\mathrm{K}$ and J-K star counts in the Baade window to $\mathrm{K}=13.5$.

\section{Results}

By comparing model predictions with RT data towards the Baade Window and RH data at three latitudes we found that :

The luminosity function most appropriate for the bulge population is similar to the disc one and quite different from the globular cluster LF for the stars of absolute magnitude between -1 and 4 . However this property of the bulge is no more true at latitudes larger than $20^{\circ}-25^{\circ}$ (ex: fig. 2) where a M3 LF is more suitable. This is probably related to the specific process of chemical evolution in the bulge. The 


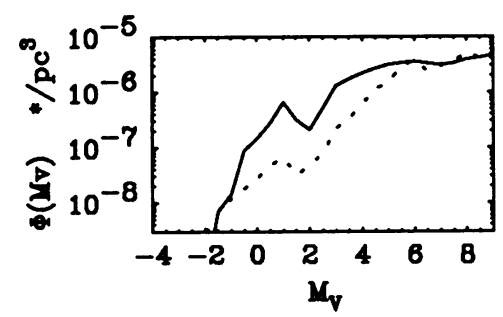

Fig. 1. Two possible bulge luminosity functions. Solid line: disc LF normalized to $0.2 \%$ of the disc; Dashed line: M3 LF.

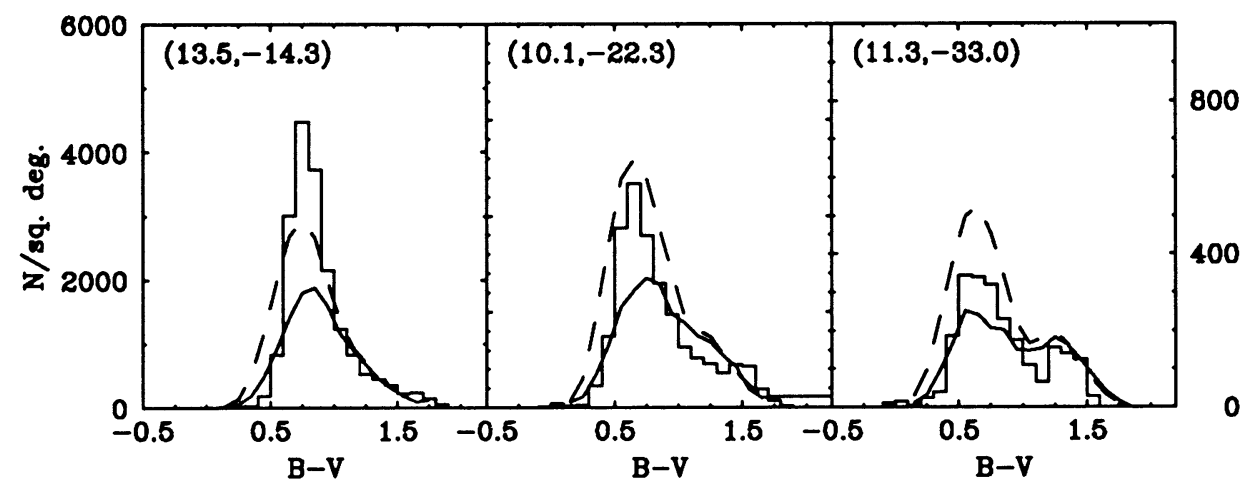

Fig. 2. B-V distribution at three latitudes for stars with $18<V<19$, compared with model predictions assuming two bulge LF. Solid line: M3 LF, dashed line: disc-like LF.

overall process of evolution may result in a luminosity function closer to the disc one than to the halo one.

The bulge density law is well fitted by a power law with an exponent of 3.8 at latitude $4^{\circ}$ (Baade window) while a smaller value $(3.1$, as the halo) is suitable for fields at $b \geq 10^{\circ}$.

This first analysis result in a bulge with a disc-like LF but a density law much steeper in the center. In the near future we plan to apply this model of population synthesis to proper motion and radial velocity distributions to contrain the kinematics of the bulge, hence to undertand more about its scenario of formation.

\section{References}

Bienaymé, O., Robin, A.C., Crézé, M., 1987, A\&A 180, 94

Robin, A.C., Crézé, M. 1986, A\&A 157, 71

Rocca-Volmerange et al., 1981, A\&A 104, 177.

Rodgers, A.W., Harding, P., 1989, A.J., 97, 1036 (RH)

Ruelas-Mayorga, R.A., Teague, P.F., 1992, A\&A suppl. 93, 61 (RT)

Yoshii, Y., Rodgers, A.W., 1989, A.J., 98, 853 